\title{
Prévalence et aspects lésionnels de I'hydatidose chez les dromadaires et les petits ruminants au nord de la Mauritanie
}

\author{
C.B. Ould Ahmed Salem ${ }^{1 *}$ F. Schneegans ${ }^{2}$ \\ J.Y. Chollet ${ }^{2}$ M.H. Jemli ${ }^{3}$
}

Dromadaire - Petit ruminant Echinococcus granulosus Hydatidose - Morbidité - Mauritanie.

\section{Résumé}

Cette étude a concerné la prévalence de l'échinococcose au nord de la Mauritanie. Le taux de prévalence de 37 p. 100, relevé chez les dromadaires originaires de la région de Zoairate, a été significativement plus élevé ( $p<0,002)$ que celui de 26 p. 100 obtenu chez les dromadaires de Nouadhibou. En revanche, dans ces mêmes régions, il n'y a pas eu de différences significatives $(p<0,4)$ entre les taux de prévalence chez les petits ruminants, avec respectivement 5,6 et 4,2 p. 100 chez les ovins, et 3,9 et 7,2 p. 100 chez les caprins. Le taux de fertilité des kystes hydatides a été dans les deux régions respectivement de 69 et 73 p. 100 chez les dromadaires, 41,2 et 42 p. 100 chez les ovins, et 35,7 et 34 p. 100 chez les caprins. Il a été significativement plus élevé $(p<0,0001)$ chez les dromadaires que chez les petits ruminants. L'infestation hydatique a globalement été caractérisée par la prédominance des localisations pulmonaires chez les dromadaires et hépatiques chez les petits ruminants. Contrairement aux petits ruminants, l'histologie des kystes hydatiques camelins a montré une structure nette avec une cuticule et une membrane proligère bien développées. Les différences entre les taux de prévalence, de fertilité des kystes hydatiques, la diversité des sites de l'infestation, et la structure histologique observée chez les dromadaires, les ovins et les caprins étaient probablement dues à la diversité des génotypes d'Echinococcus granulosus.
1. Inrsp, BP 695, Nouakchott, Mauritanie.

2. Cnerv, Nouakchott, Mauritanie.

3. ENMV, Sidi Thabet, Tunisie.

* Adresse pour la correspondance

E-mail : cheikhbaba2002@yahoo.fr

\section{INTRODUCTION}

L'échinococcose larvaire à Echinococcus granulosus, ou hydatidose, est une affection parasitaire non contagieuse, à caractère infectieux et inoculable, due au développement dans divers organes, notamment le foie et les poumons, de nombreux mammifères hôtes intermédiaires, y compris l'homme, de larves vésiculaires de type échinocoques. Le ver adulte colonise l'intestin grêle de carnivores qui en sont les hôtes définitifs (18).

En Mauritanie, les dromadaires sont des hôtes intermédiaires très réceptifs ; ils se contaminent généralement aux pâturages et développent dans leurs poumons et plus rarement dans leurs foies des kystes hydatiques qui deviennent fertiles, voire très fertiles, et potentiellement infestants pour le chien. C'est en consommant des viscères de dromadaires et éventuellement de petits ruminants parasités que le chien s'infeste par le ténia échinocoque $(3,6)$. 
Cette zoonose cosmopolite représente dans de nombreuses régions du monde un véritable fléau pour l'élevage et la santé publique (4, 19). En Mauritanie, elle est présente dans presque tous les abattoirs, mais il semble que les principales zones d'élevage camelin constituent des sites probables de foyers d'hydatidose, du fait que le dromadaire est l'hôte de prédilection pour les larves d'Echinococcus granulosus dans ce pays, même si les autres espèces sont également concernées. La région de Nouadhibou, située dans le nord-ouest du pays, grande agglomération urbaine drainant toutes les zones d'élevage du Nord, deuxième lieu d'abattage des dromadaires après Nouakchott, est approvisionnée par les dromadaires provenant de Zoairate.

Une étude lésionnelle de cette parasitose a été menée chez les dromadaires et les petits ruminants au nord de la Mauritanie afin de déterminer la prévalence de cette maladie et d'évaluer la situation épidémiologique dans cette région.

\section{MATERIEL ET METHODES}

L'étude s'est déroulée à l'abattoir de Nouadhibou. Au total, 357 carcasses de dromadaires, 128 d'ovins et 126 de caprins ont fait l'objet d'inspection post mortem pour la recherche systématique de lésions hydatiques dans les viscères. Lors de l'examen ante mortem, l'origine, l'âge et le sexe ont été notés pour chaque animal.

Au total, 166 kystes hydatiques dont 95 de dromadaires, 37 d'ovins et 34 de caprins ont été examinés. Dans le cas de saisies partielles d'organes parasités, les kystes hydatiques ont été prélevés dans leur intégralité à partir de l'organe infesté. En revanche, dans le cas d'une saisie totale, le kyste a été récupéré totalement au laboratoire. Chaque kyste a été vidé complètement de son liquide par aspiration à l'aide d'une seringue. Le contenu a été recueilli dans un flacon afin d'examiner la fertilité des kystes et la viabilité des protoscolex. Le volume des kystes, le nombre de kystes par organe et leur localisation (pulmonaire ou hépatique) ont été aussi relevés pour chaque espèce.

Pour chaque kyste, quelques gouttes de liquide hydatique ont été examinées sous microscope pour vérifier la présence ou non de protoscolex. Tous les kystes qui contenaient du sable hydatique ont été considérés fertiles, et ceux qui n'en contenaient pas comme stériles ou acéphalocystes $(7,9)$.

Une goutte de liquide hydatique fertile mélangée avec une goutte de violet de gentiane 1 p. 1000 a été placée entre lame et lamelle. Après quelques minutes, le mélange a été observé au microscope. Tous les protoscolex qui on retenu la coloration ont été considérés comme morts, et ceux qui ne l'on pas retenue comme viables (5).

Les coupes histologiques des kystes hydatiques ont été réalisées et colorées à l'hématoxyline-éosine selon la technique décrite par Saad (15).

Tous les résultats ont fait l'objet d'une analyse statistique avec le test du chi-carré.

\section{RESULTATS}

Des différences ont été observées selon l'origine géographique des animaux (figure 1). Chez les dromadaires, la prévalence de l'hydatidose des animaux originaires de la région de Zoairate (37 p. 100) a été significativement plus importante $(\mathrm{p}<0,002)$ que celle des animaux de Nouadhibou (26 p. 100). Chez les ovins, la prévalence a été de 5,9 p. 100 pour ceux de Zoairate et de 4,2 p. 100 pour les autochtones. Chez les caprins, elle a été respectivement de 3,9 et 7,2 p. 100. Il n'y a pas eu de différence significative entre les deux régions $(\mathrm{p}<0,4)$.
Les prévalences selon l'âge, le sexe et en fonction de l'espèce animale sont présentées dans les figures 2, 3 et 4. Les animaux âgés de moins d'un an n'étaient pas infestés. L'infestation était proportionnelle à l'âge des animaux : plus ils étaient âgés, plus la prévalence était élevée. La prévalence n'a pas semblé être influencée par le sexe des animaux dans les deux régions.

La localisation des kystes hydatiques est présentée dans la figure 5. L'infestation a été caractérisée globalement par la prédominance des localisations pulmonaires chez les dromadaires et hépatiques chez les petits ruminants.

Région de Nouadhibou $\square$ Région de Zoairate

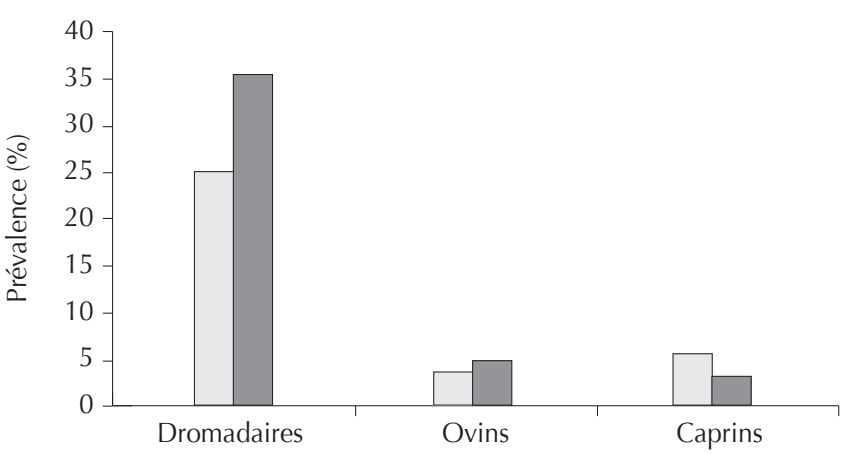

Figure 1 : taux d'infestation globale par le kyste hydatique dans les deux régions selon l'espèce animale.

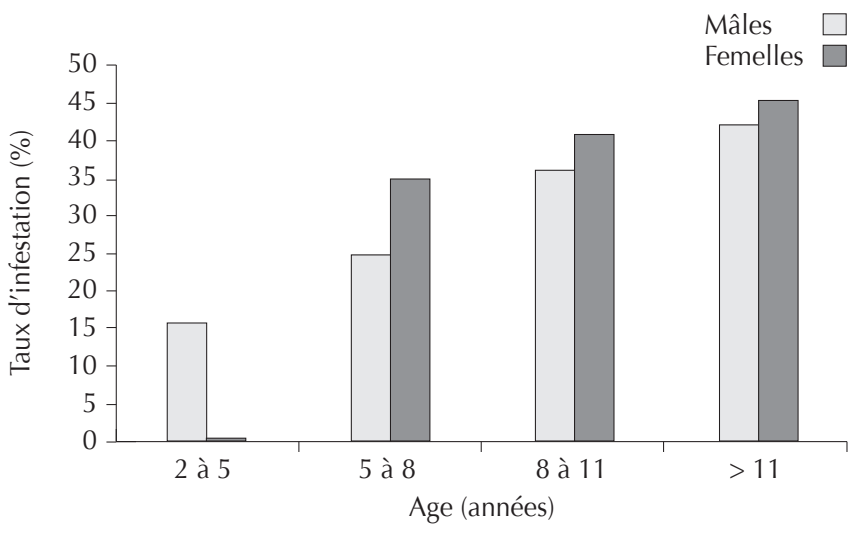

Figure 2 : taux d'infestation par le kyste hydatique chez les dromadaires selon l'âge et le sexe.

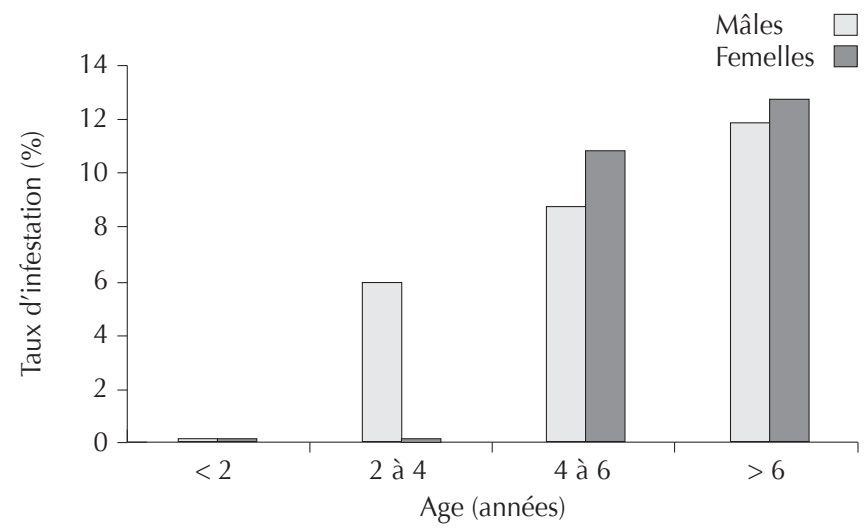

Figure 3 : taux d'infestation par le kyste hydatique chez les ovins selon l'âge et le sexe. 


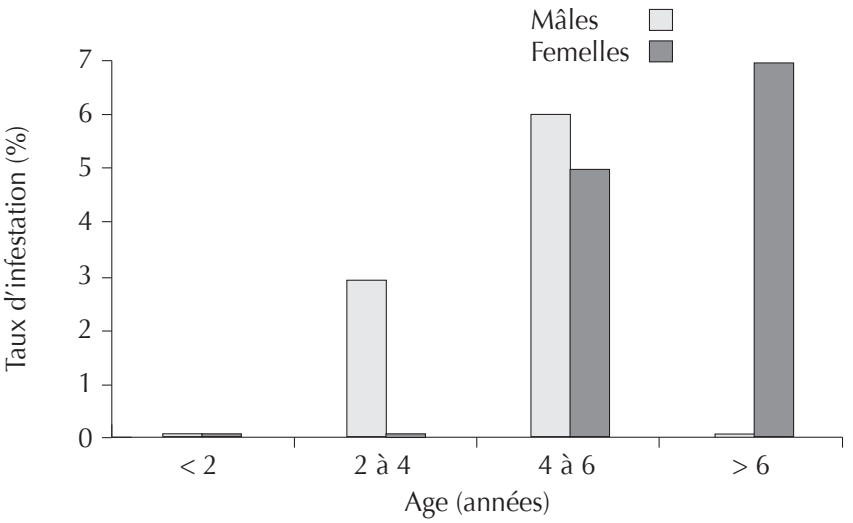

Figure 4 : taux d'infestation par le kyste hydatique chez les caprins selon l'âge et le sexe.

Le taux de fertilité a été significativement plus élevé $(\mathrm{p}<0,0001)$ respectivement dans les deux régions chez les dromadaires (69 et 73 p. 100) que chez les ovins $(41,2$ et 42 p. 100) et les caprins $(35,7$ et 34 p. 100$)$

Les caractéristiques des lésions hydatiques selon les espèces animales sont regroupées dans le tableau I. Le nombre moyen de kystes hydatique a varié entre 2 et 3 . Le pourcentage de kystes hydatiques calcifiés au niveau des poumons a été relativement faible (9 p. 100) chez les dromadaires, mais très élevé

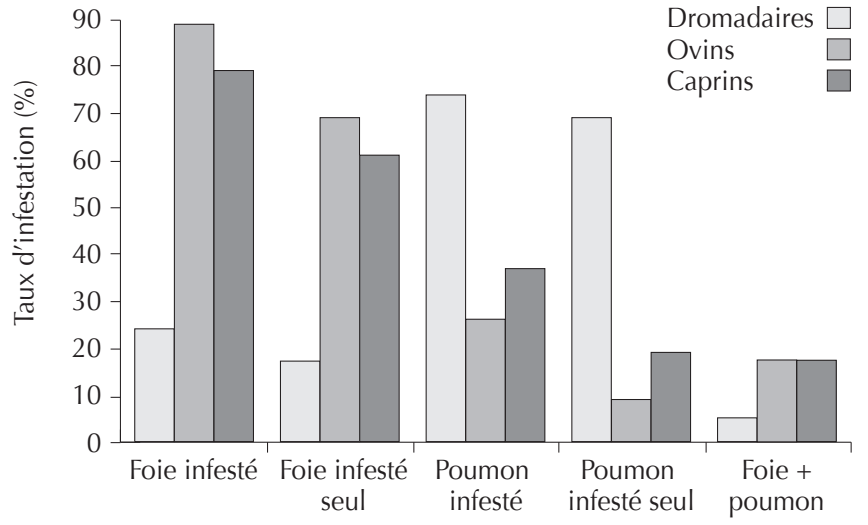

Figure 5 : localisation des kystes hydatiques dans les organes parasités selon l'espèce animale.

$(54,5$ p. 100) chez les ovins. Chez les dromadaires, les kystes ont été le plus souvent volumineux avec des éléments structuraux, comme la cuticule et la membrane proligère, bien développés. Chez les petits ruminants, ils ont été de petites tailles, avec des structures anatomiques moins nettes et le plus souvent calcifiées ou stériles.

Le nombre moyen de protoscolex a varié de 78 à 271 par millilitre selon l'espèce animale. La viabilité des protocoscolex a généralement été très élevée (tableau II).

\section{Tableau I}

Caractéristiques des kystes hydatiques chez les dromadaires et les petits ruminants

\begin{tabular}{lcccccc} 
Espèce & Organe parasité & $\begin{array}{c}\text { Nb. total } \\
\text { KH examinés }\end{array}$ & $\begin{array}{c}\text { Nb. moyen } \\
\text { KH/organe }\end{array}$ & $\begin{array}{c}\text { Nb. total } \\
\text { KH fertiles }\end{array}$ & $\begin{array}{c}\text { Nb. total } \\
\text { KH stériles }\end{array}$ & $\begin{array}{c}\text { Nb. total } \\
\text { KH calcifiés }\end{array}$ \\
\hline Dromadaire & Foie & 71 & 2 & $49(69 \%)$ & $9(12,7 \%)$ & $16(22,5 \%)$ \\
\multirow{2}{*}{ Ovin } & Poumons & 24 & 3 & $172(77,8 \%)$ & $30(13,6 \%)$ & $20(9 \%)$ \\
& Foie & 19 & 2 & $8(42,1 \%)$ & $5(26,5 \%)$ & $7(36,8 \%)$ \\
Caprin & Poumons & 11 & 3 & $3(27,3 \%)$ & $4(36,4 \%)$ & $6(54,5 \%)$ \\
& Foie & 17 & 3 & $5(29,4 \%)$ & $3(17,7 \%)$ & $6(35,3 \%)$ \\
& Poumons & 12 & 3 & $4(33,3 \%)$ & $2(19,7 \%)$ & $3(25 \%)$
\end{tabular}

$\mathrm{KH}$ : kystes hydatiques

\section{Tableau II}

Taux de viabilité des protoscolex des kystes hydatiques des dromadaires et des petits ruminants

\begin{tabular}{lccccc} 
Espèce & Organe parasité & $\begin{array}{c}\text { Vol. moyen } \\
\text { des } \mathbf{K H}(\mathbf{m l})\end{array}$ & $\begin{array}{c}\text { Nb. moyen des } \\
\text { protoscolex/ml }\end{array}$ & $\begin{array}{c}\text { Nb. moyen de } \\
\text { protoscolex vivants/ml }\end{array}$ & $\begin{array}{c}\text { \% viabilité des } \\
\text { protoscolex/ml }\end{array}$ \\
\hline Dromadaire & Foie & 10,8 & 134 & 128 & 95 \\
& Poumons & 14,1 & 271 & 217 & 80,1 \\
Ovin & Foie & 7,5 & 87 & 63 & 72,4 \\
& Poumons & 5,2 & 85 & 52 & 61,2 \\
Caprin & Foie & 5,1 & 182 & 150 & 82,4 \\
& Poumons & 5,4 & 78 & 57 & 73,1
\end{tabular}


En ce qui concerne l'histologie, les kystes hydatiques camelins ont été constitués dans la plupart des cas d'une structure conforme à celle décrite dans la littérature, avec une cuticule et une membrane proligère visibles et nettes. Comme pour les kystes hydatiques fertiles, les protoscolex étaient attachés à la membrane proligère sous forme de capsules proligères (figure 6). Pour les ovins et les caprins, la structure histologique des kystes hydatiques n'était pas typique, la cuticule et la membrane proligère étaient beaucoup moins développées. En revanche, au niveau de l'histopathologie les réactions cellulaires inflammatoires et les nécroses ont été plus fréquentes et intenses (figure 7).

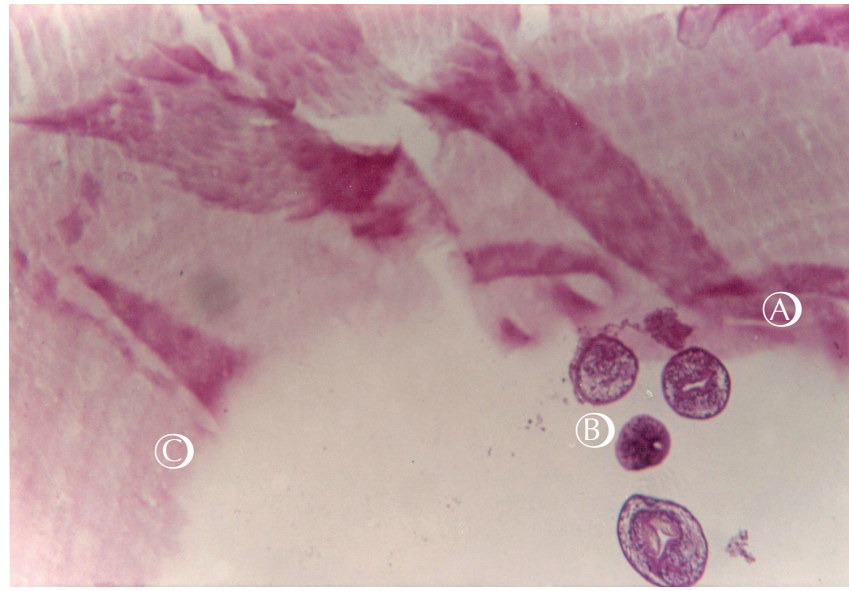

Figure 6 : coupe histologique d'un kyste hydatique pulmonaire fertile d'origine cameline ; (A) membrane germinative, (B) protoscolex et (C) cuticule (hématoxyline-éosine x 100).

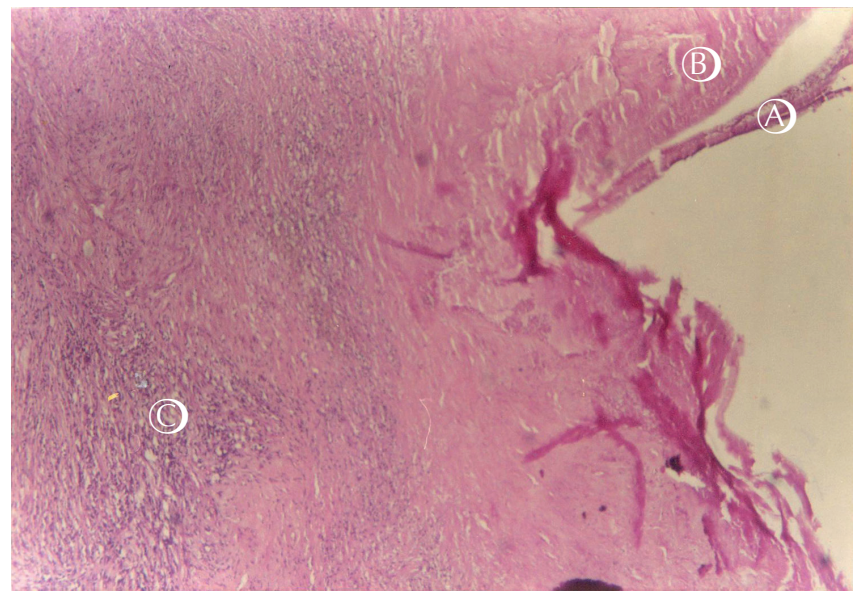

Figure 7 : coupe histologique d'un kyste hydatique stérile du foie d'origine ovine; (A) membrane germinative, (B) cuticule et (C) nécrose des tissus hépatiques (hématoxylineéosine $x$ 100).

\section{- DISCUSSION}

Dans cette étude, les auteurs ont privilégié l'inspection des carcasses d'animaux dans les abattoirs, car elle est considérée comme la technique de référence pour le dépistage de l'hydatidose chez les animaux, les épreuves sérologiques se heurtant aux problèmes de sensibilité, de spécificité et de réactions croisées $(14,20)$. La représentativité des animaux abattus n'était pas complète dans la mesure où des abattages clandestins avaient parfois lieu et l'inspection des abats était insuffisante parfois.

Les résultats de prévalence moyenne chez les dromadaires ont été inférieurs à ceux obtenus en Mauritanie $(53,07$ p. 100) (13), au Maroc (80 p. 100) (13), en Algérie (56,5 p. 100) (17), au Niger (55 p. 100) (1) et au Soudan (42 p. 100) (16), mais proches de ceux obtenus en Libye (31,9 p. 100) (12) et en Tunisie (7 à 62 p. 100) (11). Les dromadaires ont été significativement $(\mathrm{p}<0,0001)$ infestés par les kystes hydatiques dans les deux régions par rapport aux petits ruminants. Compte tenu du mode d'élevage extensif du dromadaire dans ces deux régions, on peut émettre l'hypothèse selon laquelle il y jouerait un rôle majeur dans la dissémination de l'échinococcose.

Le taux d'infestation a augmenté progressivement avec l'âge des animaux dans les deux régions, avec des différences significatives chez les dromadaires $(\mathrm{p}<0,002)$, les ovins $(\mathrm{p}<0,06)$ et les caprins ( $p<0,0001)$. Ces observations ont été rapportées chez les moutons dans le Maghreb par d'autres auteurs $(5,11)$. La prévalence plus élevée chez les animaux âgés serait due à certains facteurs socioécologiques.

Dans les deux régions, les conditions climatiques (faible pluviométrie, de l'ordre de $50 \mathrm{~mm}$ par an, et basse température) favorisent l'apparition d'une couverture végétale à base d'herbes halophytes appréciées par le dromadaire. En outre, cette zone constitue un habitat privilégié pour le chacal et autres carnivores sauvages (2, 10). Les dromadaires s'infesteraient par les œufs du ténia échinocoque, au moment des transhumances, à travers les différents pâturages et les points d'eau, comme les mares et les marigots, fréquentés par les carnivores sauvages et les chiens de bergers (17). De plus, en zone urbaine, le chien contribue aussi à l'infestation des animaux, surtout celle des petits ruminants et des troupeaux de dromadaires gardés aux entourages des agglomérations urbaines. Cela expliquerait la prévalence élevée chez le dromadaire.

L'étude lésionnelle des kystes hydatiques a montré une prédominance des localisations pulmonaires chez les dromadaires. Cette prédominance pourrait s'expliquer par le trajet de migration des embryophores. En effet, ces derniers sont libérés dans la lumière intestinale, franchissent la paroi intestinale grâce à leurs crochets puis gagnent la circulation sanguine. Dans cette migration, ils rencontrent deux filtres essentiels, le foie et les poumons. En outre, le taux élevé des localisations pulmonaires pourrait s'expliquer par la possibilité qu'ils ont d'emprunter le système veineux cave et non pas la veine porte. De plus, la diversité des lignées d'Echinococcus granulosus est aussi impliquée $(8,9)$.

Le taux de fertilité des kystes hydatiques et la viabilité des protoscolex ont varié entre les trois espèces étudiées. Les kystes hydatiques chez les dromadaires ont été significativement plus fertiles $(\mathrm{p}<0,0002)$, avec des protoscolex plus viables, que ceux trouvés chez les petits ruminants.

En ce qui concerne l'histologie, les kystes hydatiques camelins ont été constitués dans la plupart des cas d'une structure conforme à celle décrite dans la littérature : cuticule épaisse, membrane proligère, capsules proligères visibles et nettes (8). Dans les kystes hydatiques fertiles, les protoscolex ont été attachés à la membrane proligère sous forme de capsules proligères et particulièrement chez les kystes pulmonaires d'origine cameline (figure 6). Pour les ovins et les caprins, la structure histologique des kystes hydatiques n'était pas typique, la cuticule et la membrane proligère ont été beaucoup moins développées avec la présence de réactions cellulaires inflammatoires et des nécroses plus fréquentes et intenses (figure 7). Ces différents aspects lésionnels qui ont varié selon l'espèce animale étaient probablement liés à la diversité des lignées d'E. granulosus chez les différentes espèces. Ces 
résultats préliminaires concordent avec ceux observés par d'autres auteurs dans plusieurs régions du pays, d'une part, et dans certaines régions du Maghreb, en Afrique de l'Ouest et au Moyen Orient, d'autre part. Cependant, en Tunisie, certaines études sur le typage moléculaire d'isolats des lignées G1 et G6 d'E. granulosus montrent l'existence de diversités moléculaires entre les isolats camelins et ovins (12). De même, certaines études menées en Afrique de l'Ouest et au Moyen Orient signalent l'existence de lignées d'E. granulosus potentiellement infectante pour les dromadaires par rapport aux autres espèces (16).

\section{CONCLUSION}

Cette étude a montré que la prévalence de kystes hydatiques a été élevée chez le dromadaire. Ces kystes étaient caractérisés par leur structure (membrane proligère, cuticule et sable hydatique) bien visible à l'œil nu, l'importance de leur volume, leur fertilité et surtout leur localisation pulmonaire. En revanche, chez les petits ruminants, les kystes étaient beaucoup moins développés, voire calcifiés. Ces résultats préliminaires suggèrent que ces différences entre les kystes hydatiques chez les dromadaires et chez les petits ruminants seraient liées aux diversités des lignées d'E. granulosus.

Il serait donc nécessaire, compte tenu de l'importance économique, sanitaire et médicale de cette zoonose, de poursuivre et d'approfondir cette étude par la caractérisation moléculaire et la comparaison d'isolats de kystes hydatiques chez les différents animaux entre eux, d'une part, et avec des lignées de référence, d'autre part, afin d'identifier la ou les lignées d'Echinococcus granulosus circulants. Une des conséquences permettrait de préciser le rôle épidémiologique des différents animaux dans la transmission de l'hydatidose dans la région.

\section{BIBLIOGRAPHIE}

1. ARENE E.O.I., 1985. Prevalence of hydatidosis in domestic livestock in the Niger. Trop. Anim. Health Prod., 17: 3-4.

2. CHRISTY P., 1989. L'élévage du dromadaire en Mauritanie. In : Atlas élevage et potentialités pastorales. Synthèses cartographiques. MaisonsAlfort, France, Cirad-lemvt, 27 p.

3. DAVID H., WEN Y., TIAOYING L., YONGFU X., XINGWANG C.H., YAN H., YUN YANG Q.W., JIAMIN Q., 2006. Control of hydatidosis. Parasitol. int., 55: 247-252.

4. DEVELOUX M., 1996. L'hydatidose en Afrique : aspect épidémiologique. Méd. trop, 56 : 177-183.

5. EKERT J., GEMMELL M.A., MATAYS Z., SOULSBY J.L., 1984. Directive pour la surveillance et la prévention de l'echnococcose/hydatidose et la lutte contre ces maladies. Genève, Suisse, OMS, 147 p.

6. ELAYOUBI F.A., CRAIG P.S., 2004. Echinococcus granulosus coproantigens: chromatographic fractionation and characterization. Parasitology, 128: 455-465.

7. ELISSONDO M.C., ALBANI C.M., GENDE L., EGUARAS M., DENEGRI G., 2008. Efficacy of thymol against Echinococcus granulosus protoscoleces. Parasitol. int., 57: 185-190.

8. HIRIS A., HEATH D.D., LAWRENCE J.B., SHAW R.J., 1989. Echinococcus granulosus ultrastructure of epithelial changes during the first eight days of metacestode development in vitro. Int. J. Parasitol., 19: 221-229.

9. HOUIN R., FLISSER A., LIANCE M., 1994. Cestodes larvaires. Paris, France, Editions techniques, Encyclopédie médicale, 1022 p.

10. JIAO W., CHENG F., QUN Q., NURBEK, XU S.D., SUN L.F., HAN X.K., MUHAN, HAN L.L., IRIXIATI, JIE P., ZHANG K.J., ISLAYIN, CHAI J.J., 2005. Epidemiological evaluations of the efficacy of slow-released praziquantel-medicated bars for dogs in the prevention and control of cystic echinococcosis in man and animals. Parasitol. int., 54: 231-236.

11. LAHMAR S., KILANI M., TORGERSON P.R., 2001. Frequency distributions of Echinococcus granulosus and other helminths in stray dogs in Tunisia. Ann. trop. Med. Parasitol., 95: 69-76.
12. OUDNI-MRAD M., CABARET J., MRAD S., BOUZID W., MEKKI M., BELGUITHE M., SAYADI T., NOURI A., LAHMAR S., AZAIEZ R., MEZHOUD H., BABBA H., 2006. Genetic differences between Tunisian camel and sheep strains of the cestode Echinococcus granulosus revealed by SSCP. Parasite, 13: 131-136.

13. PANGUI L.J., OULD AHMEDOU E., 1996. Incidence de I'hydatidose du dromadaire en Mauritanie. Bulletin de liaisons, Association internationale francophone pour l'étude des relations environnement santé publique vétérinaire. Toulouse, France, Ecole nationale vétériniare, p. 1-12.

14. PETER M.S., 2006. Progress in diagnosis, treatment and elimination of echinococcosis and cysticercosis. Parasitol. int., 55: 7-13.

15. SAAD M.B., ZIEN ELDIN E.A., TAG EL DIN M.H., 1983. Some observation on the prevalence and pathology of hydatidosis in Sudanese camels (Camelus dromedarius). Revue Elev. Méd. vét. Pay trop., 36: 359363.

16. SEYED M.S., 2006. Present situation of echinococcosis in the Middle East and Arabic North Africa. Parasitol. int, 55: 197-202.

17. STAMATAKOS M., SARGEDI C., STEFANAKI C.H., SAFIOLIAS C. MATTHAIOPOULOU I., SAFIOLIAS M., 2009. Anthelminthic treatment: an adjuvant therapeutic strategy against Echinococcus granulosus. Parasitol. int, 58: 115-120.

18. TORGERSON P.R., HEATH D.D., 2003. Transmission dynamics and control options for Echinococcus granulosus. Parasitology, 127: 143-158.

19. YANG Y.R., ROSENZVIT M.C., ZHANG L.H., ZHANG J.Z., MACMANUS D.P., 2005. Molecular study of Echinococcus in WestCentral China. Parasitology, 131: 547-555.

20. YASIHITO S., MINORI N., KAZUHIRO N., HIROSHI Y., AKIRA I., 2006. Recombinant antigens for serodiagnosis of cysticercosis and echinococcosis. Parasitol. int., 55: 69-73.

Accepté le 25.05.2011 


\section{Summary}

Ould Ahmed Salem C.B., Schneegans F., Chollet J.Y., Jemli M.H. Prevalence and lesional aspects of hydatidosis in onehumped camels and small ruminants in Northern Mauritania

This study concerned the prevalence of echinococcosis in Northern Mauritania. The 37\% prevalence rate recorded in camels from the region of Zoairate was significantly higher $(p<0.002)$ than that of $26 \%$ obtained in camels in Nouadhibou. However, in these regions, there were no significant differences $(p<0.4)$ between prevalence rates in small ruminants, with 5.6 and $4.2 \%$ in sheep, and 3.9 and $7.2 \%$ in goats, respectively. The fertility rates of hydatid cysts were 69 and $73 \%$ in camels, 41.2 and $42 \%$ in sheep, and 35.7 and $34 \%$ in goats in the two regions, respectively. It was significantly higher $(p<0.0001)$ in camels than in small ruminants. The hydatid infestation was generally more predominant in the lungs of the camels and in the liver of small ruminants. Unlike in small ruminants, the histology of camel hydatid cysts showed a well-defined structure with a cuticle and a welldeveloped proligerous membrane. The differences between the prevalence rates, the fertility of hydatid cysts, the diversity of infestation sites, and the histological structure observed in camels, sheep and goats were probably due to different genotypes of Echinococcus granulosus.

Keywords: Dromedary - Small ruminant - Echinococcus granulosus - Hydatidosis - Morbidity - Mauritania.

\section{Resumen}

Ould Ahmed Salem C.B., Schneegans F., Chollet J.Y., Jemli M.H. Prevalencia y aspectos lesiónales de la hidatidosis en los dromedarios y los pequeños rumiantes en el norte de Mauritania

El presente estudio concernió la prevalencia de la equinococosis en el norte de Mauritania. La tasa de prevalencia de $37 \%$, encontrado en los dromedarios originarios de la región de Zoairate, fue significativamente más alto $(p<0,002)$ que el de $26 \%$ obtenido en los dromedarios de Nouadhibou. En estas mismas regiones, por el contrario, no hubo diferencias significativas $(p<0,4)$ entre las tasas de prevalencia en los pequeños rumiantes, con 5,6 y $4,2 \%$ en los ovinos, y 3,9 y $7,2 \%$ en los caprinos respectivamente. Las tasas de fertilidad de los quistes hidatidos en las dos regiones fue respectivamente de 69 y $73 \%$ en los dromedarios, 41,2 y $42 \%$ en los ovinos, y 35,7 y $34 \%$ en los caprinos. Fue significativamente más elevado $(p<0,0001)$ en los dromedarios que en los pequeños rumiantes. La infestación hidatica fue globalmente caracterizada por la predominancia de las localizaciones pulmonares en los dromedarios y hepáticas en los pequeños rumiantes. Contrariamente a los pequeños rumiantes, la histología de los quistes hidaticos camélidos mostró una estructura nítida, con una cutícula y una membrana proligena bien desarrolladas. Las diferencias entre las tasas de prevalencia, de fertilidad de los quistes hidaticos, la diversidad de los sitios de la infestación y la estructura histológica observada en los dromedarios, los ovinos y los caprinos, fueron probablemente debidas a la diversidad de los genotipos de Echinococcus granulosus.

Palabras clave: Dromedario - Pequeño rumiante Echinococcus granulosus - Hidatidosis - Morbosidad Mauritania. 